\title{
Occupying common ground
}

Corrected: Author Correction

\author{
Despite depressingly common misconceptions, fungi are not plants. However, the alliances made between these \\ two forms of life could be an inspiration for the research communities that study them.
}

$\mathrm{n}$ September, the Royal Botanic Gardens at Kew, London held a meeting and brought out a report entitled State of the World's Fungi (https://go.nature.com/2qYCuar). This follows on from the highly successful State of the World's Plants reports of 2016 and 2017. It may seem odd to move from plants to fungi. Despite the superficial similarity of some of their habits, fungi are not even close relatives of plants. Fungi share a common ancestor with Animalia that is far more recent than their divergence from the plant line of descent. Since the late 1960s they have been considered a separate kingdom of life, the diversity of which at least matches that of any other.

The phylogenetic relationships within the fungal kingdom are not well established and are the focus of debate. Historic approaches to classification proved difficult to apply to fungi. The 'bodies' of most fungi appear very similar, especially those of the most prominent groups: the ascomycetes and the basidiomycetes. They consist of single cells, or threads of connected cells called hyphae. Luckily for taxonomists, sex in these phyla involves the production of spores released from complex fruiting bodies. These fruiting bodies include the mushrooms and toadstools that most people think of when they hear the word 'fungus', but they have proved to be poor guides for taxonomists, with the presence of both convergent and divergent evolution leading to many erroneous family trees. To complicate matters further, fungi, while producing the fibrous polymer chitin, do not fossilize well, leaving a scant and highly incomplete record through which to trace their ancestry.

In the last few decades, the power of molecular genetics has been brought to bear on the problem, with phylogenies based on genome sequencing becoming the norm. This has sorted out many complicated relationships between fungal species but it has also exposed our previous views of the diversity of fungi as woefully inadequate. Currently there are around 144,000 named and classified species of fungi, with some 2,000 or so new ones added each year. Our best estimates suggest that there are in total 2.2-3.8 million species of fungi, six times more than there are plant species. Such a constant flow of new information fuels fluidity and disagreement in fungal taxonomy relating to broad brushstrokes as well as fine details. For example, last year a primarily American group produced a classification of fungi containing eight phyla (Microbiol. Spectr. http://doi.org/cw9z; 2017). Then this year, Estonian researchers revised that number to eighteen (Fungal Divers. 90, 135-159; 2018), by 2020 it could easily be revised again.

One advantage when studying the genomes of fungi is their relative compactness. An 'average' fungal genome is about 44 Megabases (Mb), while mammals are around 3,400 Mb and plants, 5,020 Mb. This makes sequencing whole genomes much cheaper than with other eukaryotes. As a consequence there are more fully sequenced fungi than plants and animals combined. Environmental sequencing sequencing all the DNA in a sample of air, water or soil - has shown that a large fraction of the fungi in the world have never been seen and cannot be cultured. There are now thousands of fungal species only known from DNA sequences, called 'dark taxa' in analogy to physicists' unvisible 'dark matter'.

A paper in Nature Microbiology this month brings single-cell genome sequencing to bear on such 'unculturable' fungi (Nat. Microbiol. http://doi.org/cw92; 2018). The researchers sequenced the genomes of eight fungi, all of which are unculturable by virtue of their lifestyles; seven are parasites (one of the tiny crustacean Daphnia, and the others of fellow fungi) and one, Blyttiomyces helices, is a saprophyte that lives on decaying pollen. The genomes from single cells as well as pools of multiple cells were sequenced, and these individual genome assemblies were then combined. Despite the similarities of their lifestyles the fungi were highly diverse, coming from four different taxa (or possibly more depending on the exact classification scheme used). There were, however, deficiencies in their metabolic networks, which point to the root of their unculturability. They also have higher ploidy numbers than would be anticipated, possibly related to their early divergence during fungal evolution.

Notwithstanding the evolutionary gulf between them, there are deep functional links between fungi and plants that justify their joint inclusion in botanical collections such as that at Kew. At a most superficial level, both are commonly encountered in gardens; both are, on the whole, sedentary; and let us not forget that both can be not only edible, but also delicious. We eat well over 300 species of fungus, specifically their fruiting bodies. The actual number could be much higher as most are wild scavenged; very few fungi can be grown commercially. Even so, the global market for edible fungi is estimated at around US $\$ 42$ billion per year. Yeast and related fungi also act as fermenters in the production of alcohols, bread and fermented foods, such as kefir.

Fungi are also important disease agents of plants and plant crops. Wilts, rots and cankers are all caused by fungi, while Pyricularia oryzae - also known as Magnaporthe oryzae - infection results in the devastating disease, rice blast. Also, a Fusarium oxysporum strain is responsible for Panama disease, which wiped out the Gros Michel cultivar of bananas in the 1950s and may soon do the same to the modern Cavendish variety.

Despite the deleterious effects of fungal diseases, the benefits accruing from relationships between plants and fungi far outweigh them. An extreme example are orchids, which, lacking endosperm in their seeds, can only germinate and grow if fungal hyphae are present to sustain them through their initial development. A total of $90 \%$ of living plants are associated with mycorrhizal fungi, forming intimate relationships in which the plants trade their products of photosynthesis for the fungi's soil-derived nutrients (in particular, phosphorus). The maintenance of this symbiosis requires subtle communication between fungi and plant that is yet to be fully unravelled. Earlier this month, for example, a paper in Nature Communications identified a plant-derived Ser/Thr receptor-like kinase that localizes to membranes surrounding the fungal feeding structures in mycorrhiza, called arbuscules (Nat. Commun. 9, 4677; 2018). Without this kinase, transfer of lipids to the fungus and subsequent storage is disrupted resulting in an abrupt discontinuation of their mutually beneficial relationship.

Fungi are a diverse, fascinating and substantial branch on the tree of life. Historic accident may have centred their study in botanical institutions, but bringing together the two kingdoms has been as beneficial to mycologists and phytologists as to the organisms themselves.

Published online: 5 December 2018 https://doi.org/10.1038/s41477-018-0336-1 